\title{
Biocompatibility of a droplet-mixing device using an electro-conjugate fluid
}

\author{
Kenjiro TAKEMURA*, Kiho KOBAYASHI* and Kazuya EDAMURA** \\ *Department of Mechanical Engineering, Keio University \\ 3-14-1 Hiyoshi, Kohoku-ku, Yokohama 223-8522, Japan \\ E-mail: takemura@mech.keio.ac.jp \\ **New Technology Management Co., Ltd. \\ 2-9-1-306 Higashi-shinkoiwa, Katsushika-ku, Tokyo 124-0023, Japan
}

Received 30 July 2015

\begin{abstract}
An electro-conjugate fluid (ECF) is a type of functional fluid that produces a jet flow under a non-uniform electric field. We use an ECF to generate a rotational flow in a tiny cylindrical chamber. When multiple droplets are dripped into the rotational flow of the ECF, they may be subjected to a centripetal force because of the difference in density between the droplets and ECF. This causes the droplets to move to the center of the chamber and mix with each other. In this paper, we conducted luminance measurements using a bioluminescence reaction to verify the suitability of this device for biological applications. We observed the luminescence produced by mixing droplets of luciferin and luciferase, and measured luminance maxima and area to assess the mixing ability of the device. In addition, because the enzyme luciferase can be deactivated by surroundings, we confirmed whether the proposed mixing method has any harmful influence on biological samples or not. To better understand the properties of the device, we conducted control experiments with two other set of conditions; i.e., without ECF and with still ECF. Our results confirmed that there was no difference in mixing ability between the three systems, and the influence of an electric- and centrifugal-field generated in the device on mixing might be negligible for biological applications.
\end{abstract}

Key words : $\mu \mathrm{TAS}$, Mixing, Electro-conjugate fluid, Functional fluid, Bioluminescence

\section{Introduction}

Micro total analysis systems ( $\mu \mathrm{TAS})$ are promising miniature analysis systems for medical and biochemical analyses. Droplet-based $\mu$ TAS in particular handles samples in droplet state, which reduces both the sample volume and inspection time, as well as possessing the advantages of conventional $\mu$ TAS such as simple operation, multiple functions and small size (Shoji, 1999). Unlike the way that samples and reagents flow along narrow channels with the aid of micro pumps and valves, it is difficult to transport and mix samples and reagents as droplets. Therefore, practical droplet-based $\mu$ TAS have not yet been developed, although various droplet-based methods have been developed, such as electrostatic droplet actuation (often called electrowetting on dielectric or EWOD) (Hadwen, et al., 2012, Fair, 2007), standing acoustic waves actuation (Alghane, et al., 2011, Wixforth, 2003), and local heating actuation (Kato, et al., 2013). Some of our authors developed a device that transports droplets bidirectionally in a $2 \times 2$ channel matrix using an electro-conjugate fluid (ECF) (Abe, et al., 2013). An ECF is a type of functional fluid suitable for microfluidic devices that produces a jet flow under an applied non-uniform electric field (Figure 1) (Mori, et al., 2011). The device using an ECF could mix droplets iteratively within just four short channels. However, each droplet had to be controlled to contact with another droplet at the junction of channels, and mixed one by one when multiple droplets were to be mixed. This increased the inspection time, and made the whole system bigger because it required cameras and computers apart from the device itself to realize position feedback control of droplets. Thus, previous methods have realized droplet transportation and mixing, but not maximized the advantages of droplet-based $\mu$ TAS.

Turning to the research focused on droplet mixing only, there are numerous methods to mix droplets, such as a 
technique based on EWOD (Fowler, et al., 2002) and trapping droplets in the node of ultrasonic oscillation (Nakamura, et al., 2013). In these methods, droplets can be mixed with relatively simple operations such as switching the voltage to the appropriate electrode sequentially, or varying the amplitude of voltage in a cyclic manner. However, these operations have to be executed in a timely manner.

Using the rotational flow of an ECF, which is easily generated in a minute space, we have developed a device to mix multiple droplets in a novel but simple way (Kobayashi, et al., 2013). To put this device to practical use in medical and biochemical fields, the biocompatibility of our device must be confirmed. In this paper, we conduct luminance measurements using a bioluminescence reaction to verify that biological samples in droplet state can be safely mixed using our device.

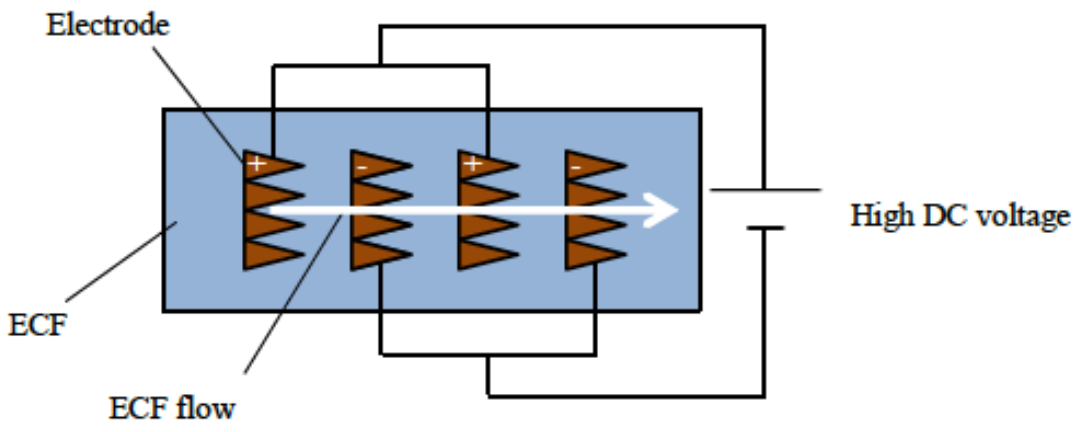

Fig. 1 Schematic diagram of ECF flow. The ECF produces a jet flow (ECF flow) under an applied non-uniform electric field.

\section{Droplet-mixing device using an electro-conjugate fluid}

Our droplet-mixing device (Kobayashi, et al., 2013) is shown in Fig. 2. The device is mainly composed of a base, substrate and floating plate. The dimensions of each component are shown in Fig. 2. The substrate, which is placed at the bottom of the chamber (formed in the base), is used to apply the voltage to generate the rotational flow of the ECF. As shown in Fig. 3, the substrate has two electrodes arranged in a radial fashion. Connecting the outer electrode to the positive terminal and inner electrode to the ground, the rotational flow is generated in anticlockwise direction. A pair of serrated and arc edges is formed between the electrodes. The dimensions of the electrode pair were determined to generate relatively active ECF flow, and have been reported previously (Yokota, et al., 2006). The floating plate lies unfixed in the chamber. When the voltage is applied to the electrodes, the floating plate floats and spins along with the

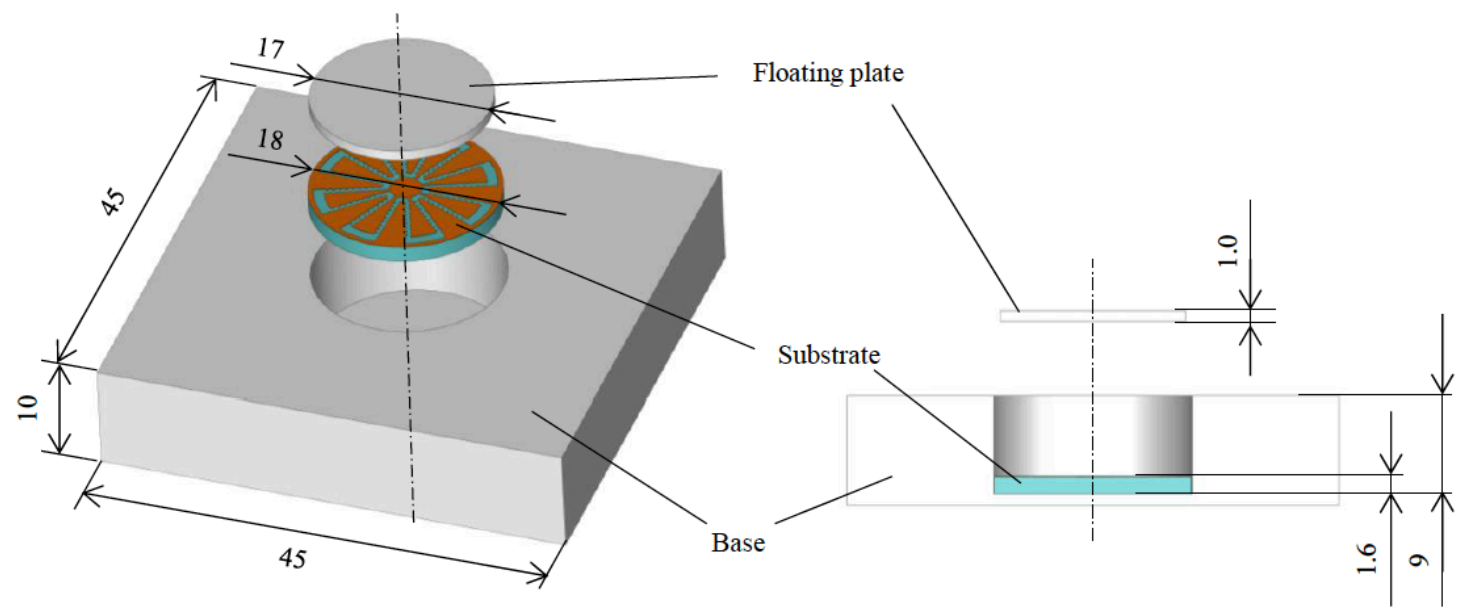

unit: mm

(a)

(b)

Fig. 2 Diagrams of mixing device (a) configuration and (b) cross section. 


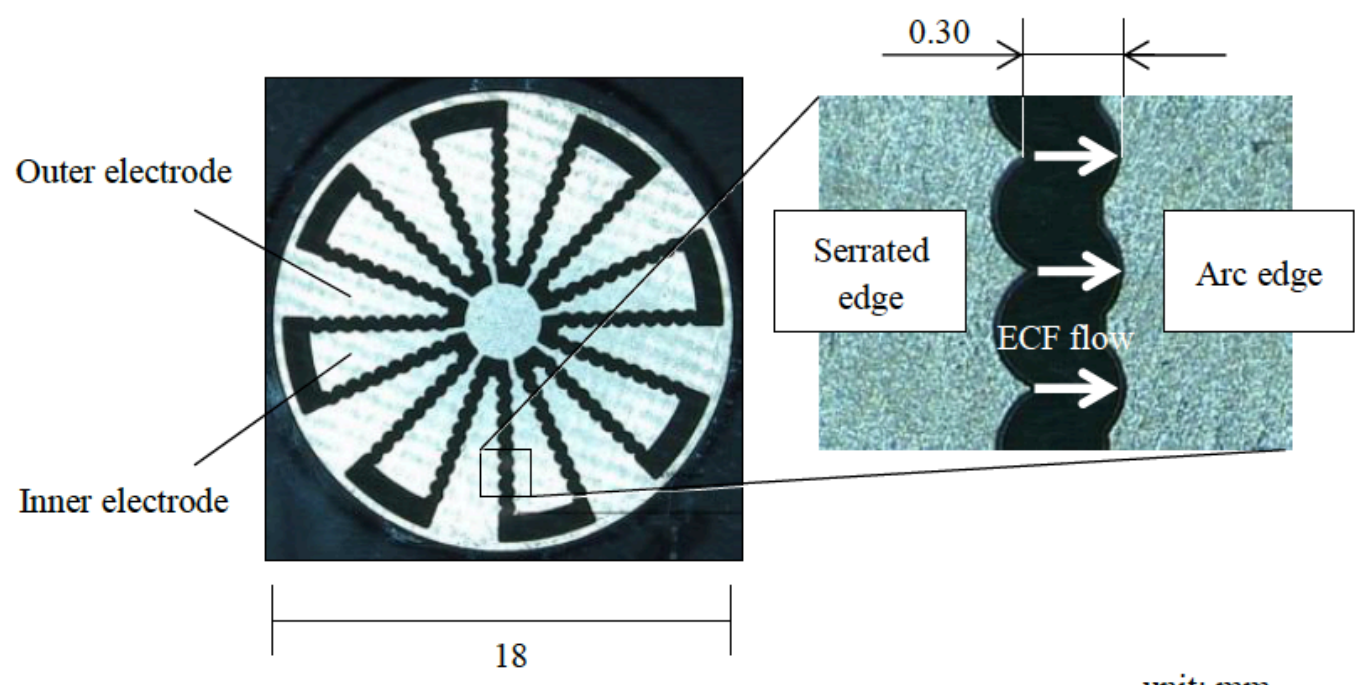

unit: $\mathrm{mm}$

Fig. 3 Substrate and electrodes. Electrodes have pairs of serrated and arc edges arranged in a radial fashion.

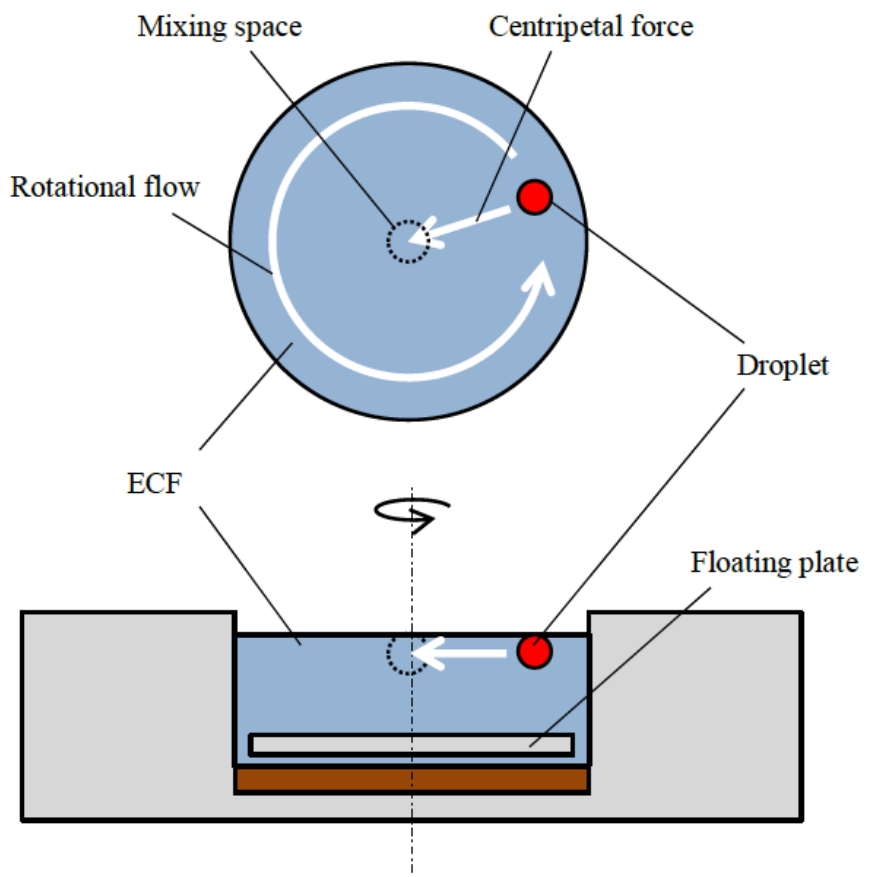

Fig. 4 Mixing method proposed in our study. When droplets are dripped into the ECF rotational flow, they may be subjected to a centripetal force caused by the difference in density between droplets and ECF, resulting in droplets moving to the center of the chamber and mixing with each other.

rotational flow of the ECF, preventing droplets from directly attaching to the substrate. Both the base and floating plate are made of polytetrafluoroethylene (PTFE) so as to have hydrophobic nature.

The mixing principles of the device are summarized as follows (Fig. 4). Simply applying a voltage to the electrodes at the same time generates rotational flow in the ECF chamber. A droplet is then dripped into the chamber, where it is subjected to a centripetal force provided because the density of the ECF is higher than that of the droplet. As a result, the droplet moves along a helical trajectory towards the center of the chamber. If another droplet is added, the two droplets may automatically mix at the center of the device. This simplicity of the mixing principle provides following virtues. First, droplets can be manipulated in non-contact and non-invasive. There are various methods which premise on the contact of the droplets with the substrate such as EWOD actuation (Hadwen, et al., 2012), magnetic actuation (Dorvee, et al., 2004, Lehmann, et al., 2006), and chemically wettability change actuation (Ichimura, et al., 2000, Khoo, 
et al., 2006) etc., however it is pointed out that those methods have risk of contamination from a practical application perspective (Basu and Gianchandani, 2007). There are also some non-contact methods like standing acoustic wave actuation (Alghane, et al., 2011, Wixforth, 2003) and optical tweezers (Kumazaki, et al., 2007), but those could lead to damage the biological samples on account of the local energy, which cannot be avoided on their principle. Thus, the advantage of non-contact and non-invasive in our device could improve safety measures in droplet-based $\mu$ TAS. Second, our device depends on neither droplets' volume nor quantity, and also property. In actual analysis, sample doesn't always react with isometric reagents one by one, though majority of mixing experiments are conducted as if it did in previous researches. Moreover, some methods require to limit the droplets' chemical characteristic (e. g., the polarized droplet must be used in the EWOD actuation), or to add some specific substances in order to change the droplets' property (e. g., magnetic particles are to be contained in magnetic actuation). Compared with those methods, our device can actuate droplets with any volume, any quantity and any property as long as they have lower density than that of the ECF. Third, the most important and unique feature is that our device can mix droplets autonomously. There is no need to detect and control droplets' position, and also it is unnecessary to align the pipette. Roughly dripping the droplets to the chamber, they surely mix. Lastly, our device has the possibility as an on-chip device. With the ECF flow generation, we do not need any external pumping systems apart from the device. For the aforementioned features, our device can be a fundamental technology which has a possibility to prevail in broad medical and biochemical community.

Because ECF is a generic term for the fluids defined in the reference (Yokota, et al., 2000), we selected the optimal one for our device, FF-505-12 (New Technology Management, Co., Ltd., Tokyo, Japan), according to the mixing principles mentioned above. FF-505-12 has relatively high density, and high water- and oil-repellency, with the highest insolubility for water-soluble matter among all ECFs. Note that the droplets used in this research are colored water and hydrosoluble biological samples. The physical properties of FF-505-12 are listed in Table 1.

Table 1 Physical properties of FF-505-12

\begin{tabular}{lc}
\hline Density $\left(\mathrm{kg} / \mathrm{m}^{3}\right)$ & 1.550 \\
Viscosity $(\mathrm{mPa} \cdot \mathrm{s})$ & 1.1 \\
Specific inductive capacity & 5.1 \\
Conductivity $(\mathrm{S} / \mathrm{m})$ & $3 \times 10^{-9}$ \\
Dielectric strength at $2.5 \mathrm{~mm}$ gap $(\mathrm{kV})$ & 27 \\
\hline
\end{tabular}

\section{Preliminary experiment}

As mentioned in section I, this study aims to verify the biocompatibility of our droplet-mixing device. This section summarizes the basic characteristics of the mixing device.

In our previous study, we determined the driving conditions suitable for droplet mixing (Kobayashi, et al., 2013). We used water droplets with a volume of $3.0 \mu \mathrm{l}$ colored in red and blue, and confirmed the effectiveness of mixing for various input voltages and ECF volumes. For an applied voltage of $1.74 \mathrm{kV}$ and ECF volume of $800 \mathrm{ml}$ (depth of 5.5

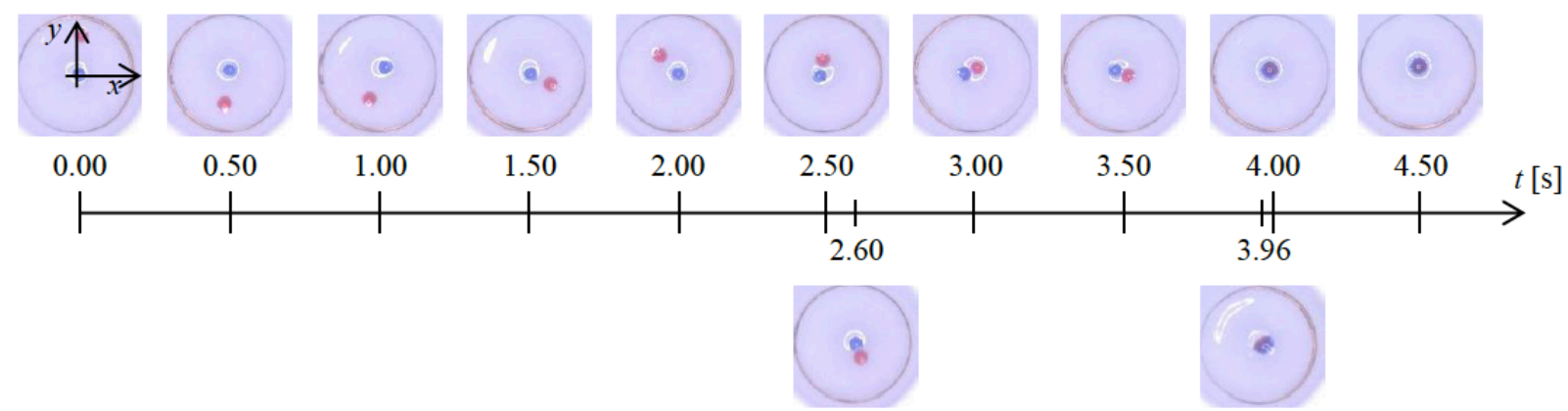

Fig. 5 Actual view of the mixing experiment: A red droplet moves spirally to the center and comes in contact with a blue droplet at $t=2.60 \mathrm{~s}$. The two droplets then roll together and mix at $t=3.96 \mathrm{~s}$. The droplets stay at the center of the device until the applied voltage is removed. 
$\mathrm{mm}$ ), two droplets successfully mixed in $3.96 \mathrm{~s}$ at the center of the chamber without any complex controller, as shown in Fig. 5.

Because this study uses biological samples that are easily affected by temperature change, we determined the temperature shift of the ECF when a rotational flow is generated in the mixing chamber. The monitoring time was 480 $\mathrm{s} ; 300 \mathrm{~s}$ with voltage applied and then $180 \mathrm{~s}$ without any voltage, because a previous cytotoxic test showed that biological samples may not be influenced by an ECF for at least 300 s (Kobayashi, et al., 2014). The ECF surface temperature was measured by a noncontact thermometer (Raynger ST6, Raytek Corporation, Santa Cruz, California, USA) placed $0.30 \mathrm{~m}$ above the ECF surface. Note that only the surface temperature of the ECF is important because the biological samples float in the ECF chamber due to the difference in density between the samples and ECF. The experimental parameters were the optimized values mentioned above; i.e., an applied voltage of $1.74 \mathrm{kV}$ and $\mathrm{ECF}$ volume of $800 \mathrm{ml}$. Because the ECF requires high DC voltage in the order of several $\mathrm{kV}$ to flow, the voltage was applied via an amplifier (HV-10P (A), Matsusada Precision Inc., Shiga, Japan). Figure 6 shows the temperature shift at the ECF surface over time. During the first $300 \mathrm{~s}$ with voltage applied, which means rotational flow was generated in the ECF chamber, the temperature slightly increased. After the voltage was removed, the ECF returned to its initial temperature. This slight change of temperature is considered to be negligible in the following bioluminescent experiment because the luminance from the luciferin-luciferase reaction is stable between 20 and $25^{\circ} \mathrm{C}$ (Endo, 2007). It is also known that the decay time of the luciferin-luciferase reaction gets slower at $30^{\circ} \mathrm{C}$ or higher (Mochizuki, et al., 2013). These results confirm that the mixing device can be used for at least $300 \mathrm{~s}$ in the luminance measurement experiment without being affected by temperature change.

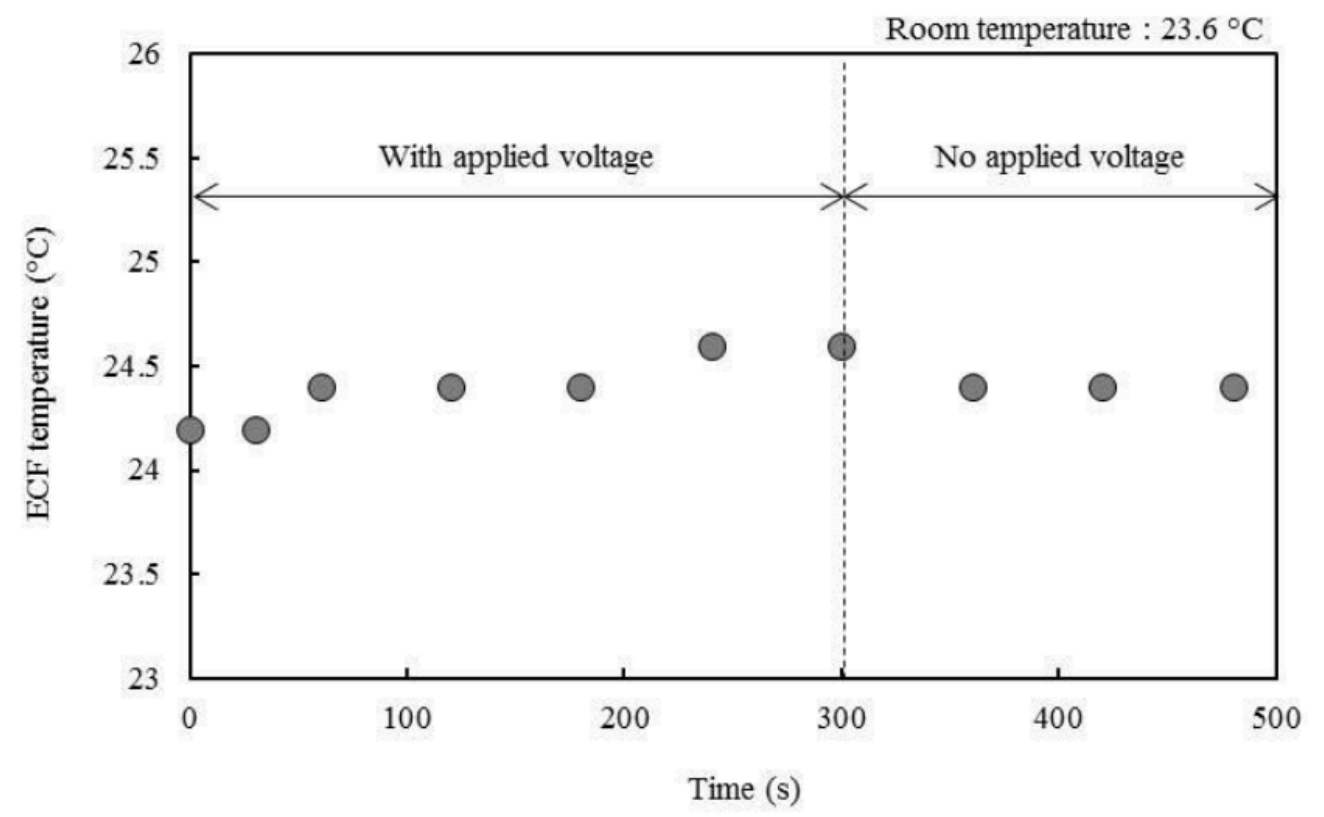

Fig. 6 Shift of ECF temperature over time. The monitoring time was set for $480 \mathrm{~s} ; 300 \mathrm{~s}$ with voltage applied and the next $180 \mathrm{~s}$ without any voltage.

\section{Bioluminescence measurement}

\subsection{Experimental purpose}

Bioluminescence is the emission of cold light from a living organism (Shimomura, 2012, Harvey, 1916). Although there are various different light-emitting reactions, they usually require two substrates, luciferin and luciferase. Luciferin is an organic compound that exists in luminous organisms and provides the energy for light emission by being oxidized, normally in the presence of a specific luciferase. Luciferase, an enzyme, catalyzes the oxidative light-emitting reaction of luciferin ( $\mathrm{Li}$, et al., 2013). Among various types of bioluminescence reactions, firefly bioluminescence has received considerable attention. Addition of ATP as a cofactor to mixtures of luciferin and luciferase results in bright, long-lasting luminescence (McElroy, 1947). This reaction can be expressed as,

$$
\text { luciferin }+\mathrm{ATP}+\mathrm{O}_{2} \stackrel{\text { luciferase }, \mathrm{Mg}^{2+}}{\longrightarrow} \text { oxyluciferin }+\mathrm{CO}_{2}+\mathrm{AMP}+\mathrm{PPi}+\text { luminance }
$$

Firefly bioluminescence has been widely used to detect ATP (Fukuda, et al., 2011) because it is simple, highly 
sensitive and quick. The Eq. 1 suggests that if there is a difference in luminance value or duration in a condition which the amount of each substance is same, it means that the reaction rate, i.e., the mixing degree makes the difference. Some previous studies defined and quantified mixing efficiency on their own way such as using a value of concentration (Wang, et al., 2008), however we can easily confirm the mixing ability by observing the luminance produced by the luciferin-luciferase reaction. Moreover, because the enzyme can be deactivated by the surrounding environments, the result of the luciferin-luciferase reaction can also gauge whether the experimental conditions have any harmful influence on biological samples or not. For these reasons, we used the firefly luciferin-luciferase reaction to examine the mixing ability and biocompatibility of our droplet-mixing device. Whether a centripetal force affects the mixing degree of a droplet positively or negatively has not been reported. Also, with regard to biocompatibility, we wish to confirm that biological samples are not adversely affected by either the ECF or electric- and centrifugal-field generated in our device.

\subsection{Procedure}

To confirm the mixing ability and biocompatibility of the droplet-mixing device, we measured the luminescence produced by luciferin and luciferase added to our device as droplets with the following three sets of experimental conditions (Figure 7): (1) Without ECF. A luciferin droplet was dripped against a luciferase droplet on a PTFE plate in the atmosphere. (2) With still ECF. Luciferin and luciferase droplets were attracted to each other by surface tension in the ECF. (3) With rotating flow of the ECF. Luciferin and luciferase droplets were mixed in our mixing device under an applied voltage. By observing the difference between the results from the conditions with still ECF (2) and with rotating flow of the ECF (3), we can confirm the effects of the centripetal force and the electric field on droplet mixing. Also, we can confirm how the ECF itself affects the enzyme luciferase by comparing the results for the conditions without ECF (1) and with still ECF (2). Each experiment was conducted in a dark box to remove any light other than the luminance produced by the reaction. Luciferin (containing ATP) and luciferase solutions (Hota-light, Kikkoman Co., Ltd., Chiba, Japan) were added as droplets with a volume of $3.0 \mu 1$ to the device. The experimental parameters were the same as those used in the preliminary experiments (Section II).

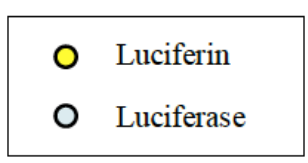

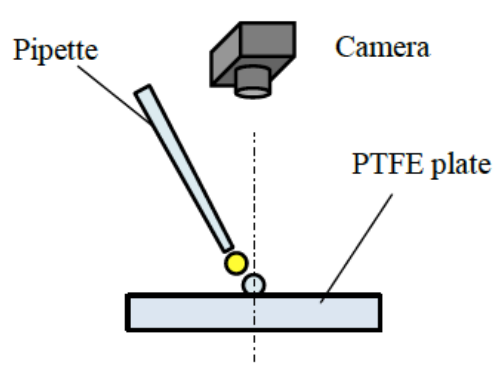

(1)

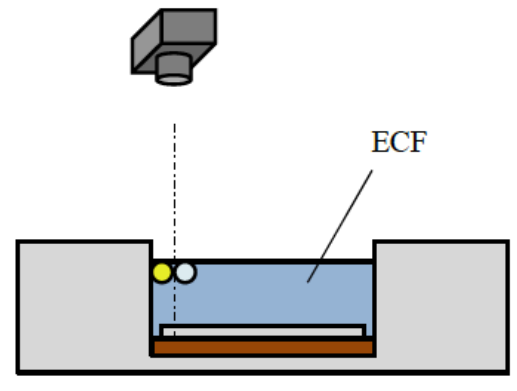

(2)

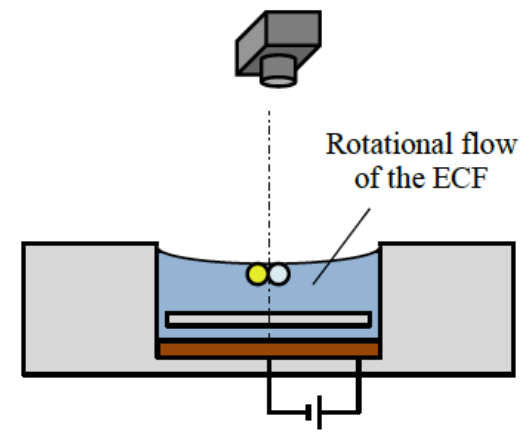

(3)

Fig. 7 Experimental conditions. (1) Without ECF, (2) with static ECF and (3) with rotating flow of the ECF

The luminescence was observed for $300 \mathrm{~s}$ at $5.0 \mathrm{~s}$ intervals. The RGB value of each still image was acquired pixel-by-pixel. Then, we converted RGB values into luminance values $Y$ according to the National Television System Committee (NTSC) standard using the following equation (Sudyoadsuk, et al., 2014). Note that the value for $Y$ is between 0 and 255 .

$$
Y=0.298912 \times R+0.586611 \times G+0.114478 \times B
$$

For each image, we then determined the luminance maxima $Y_{\max }$ and luminance area $N_{\text {area }} Y_{\max }$ is the highest luminance value among all pixels in the image, while $N_{\text {area }}$ is the number of pixels that have a luminance value higher than a set threshold. The threshold in this study was 28 , which is the maximum luminance value obtained without any 
reaction in the dark box. By comparing $Y_{\max }$ and $N_{\text {area }}$ obtained for all the sets of conditions, we can evaluate the mixing ability and biocompatibility of our droplet-mixing device using an ECF.

\subsection{Results}

The results of our luminance experiments are shown in Figs. 8 and 9. It is known that the luminance produced by the luciferin-luciferase reaction is intense initially, and gradually fades away over time (Gabriel, et al., 2014). This trend could also be seen clearly in the results. Just after two droplets came in contact with each other at $t=0 \mathrm{~s}$, both $Y_{\max }$ and $N_{\text {area }}$ decreased over time for all three conditions. Looking closely at Fig. 8, for the experiment without ECF, $Y_{\max }$ is much higher initially than for the other conditions; however, it converged to the values for the other two conditions after $t=100 \mathrm{~s}$. This may arise from the initial mixing energy. The droplet in the experiment without ECF (1) was added by means of air pressure using a pipette, so the kinetic energy of this droplet is relatively higher than those for the other conditions, resulting in effective mixing of the droplets during the initial stage. This may cause the difference of mixed states during the initial stage $(<100 \mathrm{~s})$. In other words, there is no difference between the conditions except for the initial stage. This suggests that the centripetal force does not negatively affect the mixing degree of the droplets. Also, $N_{\text {area }}$ is higher for the experiment without ECF (1) than for the other conditions (Fig. 9). This is because the droplet for the experiment without ECF was attached to the PTFE plate and was not a sphere like the droplets would be in the ECF (conditions 2 and 3). This makes the droplet area appear larger from above. In addition, the droplet area of the experiment with rotating-flow ECF (3) may become smaller due to the centripetal force. As a result, we cannot easily compare the absolute values of $N_{\text {area }}$ for the three conditions. However, it should be noted that the decrease ratio of $N_{\text {area }}$ for each condition was almost the same. This suggests that luciferin was consumed

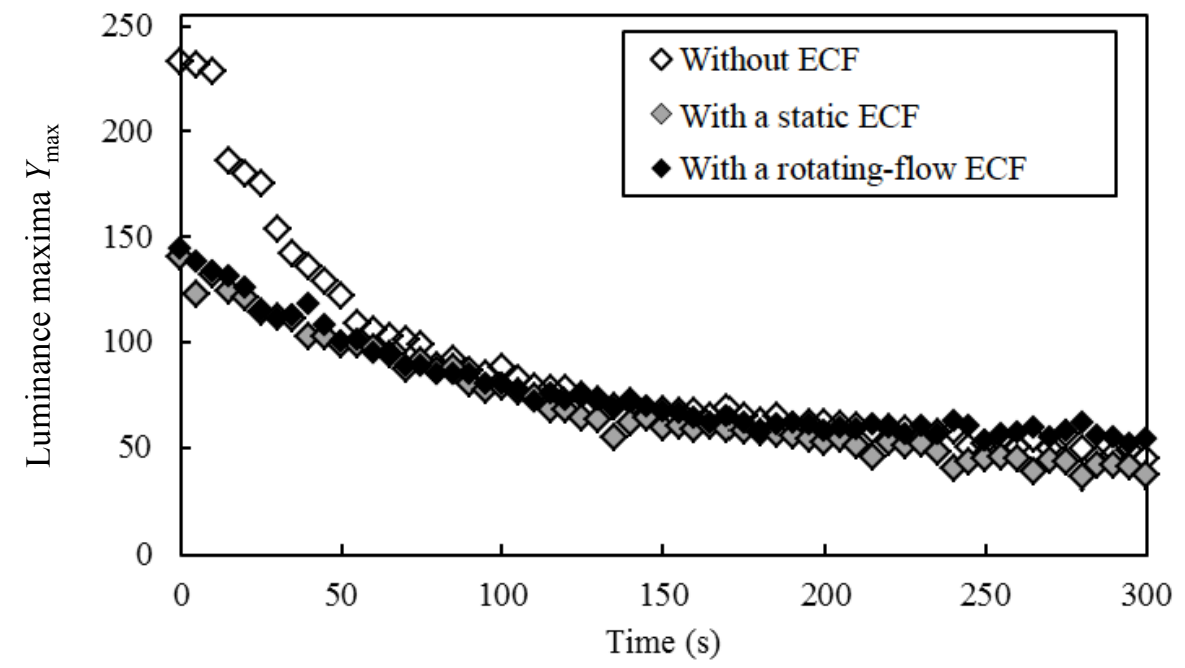

Fig. 8 Shift of luminance maxima $Y_{\max }$ over time.

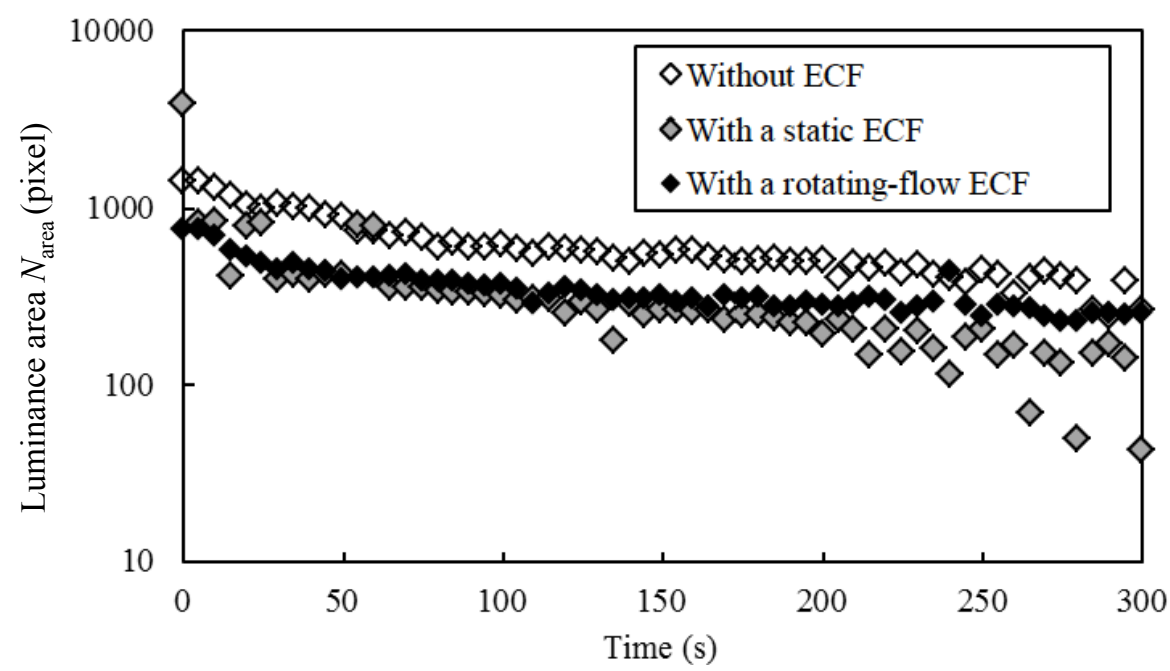

Fig. 9 Shift of luminance area $N_{\text {area }}$ over time. 
at the same rate for all of the conditions, so the luciferase in the experiment with rotating flow of the ECF (3) operates as well as in the other two conditions. Thus, it can be concluded that the electric- and centrifugal-field generated in our device does not have a harmful effect on the enzyme. However, the data with a static ECF is much largely fluctuating compared with other conditions. In this case, the droplets are in contact with the chamber wall due to the surface tension. This makes the observation difficult because of the presence of wall, i.e., it is difficult to perfectly align the wall and the optical axis of camera in parallel. As a result, the light emitted by the reaction may reflect at the wall. This might cause the variation of the data. While fluctuating, the decreasing trend can be seen in Fig. 9.

Although there is as yet a slight difference in mixing ability among all conditions, our device has a capacity to shorten the inspection time. As against the experimental condition with rotating flow of the ECF (3) which can mix droplets autonomously, the condition without ECF (1) needs pipette positioning accurately to drip the droplets at a certain place. The condition with still ECF (2) has more serious problems that it is uncertain where and even whether the droplets mix. Both operations in condition (1) and (2) are supposed to be more difficult with the decreasing volume of the droplets. Generally, diffusion time of molecules is proportional to the square of the scale length. For example, when the diameter of the droplet is to be hundredth part, the diffusion time of the molecules are in ten thousandth, which means the reaction that takes usually 1 hour can be reduced to $0.36 \mathrm{~s}$. Therefore, using our device which can easily deal with smaller droplets results in a reaction faster. This is the advantage of using the rotating flow of the ECF above other conditions.

\section{Conclusion}

In this paper, we conducted luminance experiments using a bioluminescence reaction to verify the suitability of our droplet-mixing device using an ECF for biological applications. We measured the luminescence produced by mixing luciferin and luciferase droplets under different conditions. The experimental results confirmed that our mixing device has sufficient mixing ability without having a harmful influence on biological samples.

\section{References}

Abe, Y., Takemura, K., Sato, K., Yokota, S., Edamura, K., Droplet $\mu$ TAS using electro-conjugate fluid -feedback position control of multiple droplets in flow channel matrix -, Sensors and Actuators A: Physical, Vol. 198 (2013), pp. 1-7.

Alghane, M., Chen, B. X., Fu, Y. Q., Li, Y., Luo, J. K. and Walton, A. J., Experimental and numerical investigation of acoustic streaming excited by using a surface acoustic wave device on a $128^{\circ} \mathrm{YX}-\mathrm{LiNbO} 3$ substrate, Journal of Micromechanics and Microengineering, Vol. 21, No. 1 (2011), pp. 1-11.

Basu, A. S. and Gianchandani, Y. B., A 128-pixel digitally-programmable microfluidic platform for non-contact droplet actuation using marangoni flows, Proceedings of 2007 International Solid-State Sensors, Actuators and Microsystems Conference (Transducers2007) (2007), pp. 771-774.

Dorvee, J. R., Derfus, A. M., Bhatia, S. N. and Sailor, M. J., Manipulation of liquid droplets using amphiphilic magnetic one-dimensional photonic crystal chaperones, Nature Materials, Vol. 3 (2004), pp. 896-899.

Endo, K., The Annals of Science Education Center attached to Hokkaido Education Research Institute, No. 19 (2007), pp. 108-111. (in Japanese)

Fair, R. B., Difital microfluidics: is a true lab-on-a-chip possible?, Microfluid Nanofluid, Vol. 3 (2007), pp. $245-281$.

Fowler, J., Moon, H., and Kim, C. J., Enhancement of mixing by droplet-based microfluidics, Proceedings of the fifteenth IEEE International Conference on Micro Electro Mechanical Systems (MEMS2002), (2002), pp. 97-100.

Fukuba, T., Aoki, Y., Fukuzawa, N., Yamamoto, T., Kyo, M. and Fujii, T., A microfluidic in situ analyzer for ATP quantification in ocean environments, Lab on a Chip, Vol. 11 (2011), pp. 3508-3515.

Gabriel, G. V. M. and Viviani, V. R., Novel application of $\mathrm{pH}-$ sensitive firefly luciferases as dual reporter genes for simultaneous ratiometric analysis of intracellular $\mathrm{pH}$ and gene expression/location, Photochemical \& Photobiological Sciences, No. 13 (2014) pp. 1661-1670.

Hadwen, B., Broder, G. R., Morganti, D., Jacobs, A., Brown, C., Hector, J. R., Kubota, Y. and Morgan, H., Programmable large area digital microfluidic array with integrated droplet sensing for bioassays, Lab on a Chip, Vol. 12 (2012), pp. 3305-3313. 
Harvey, E. N., Studies on bioluminescence, American Journal of Physiology, Vol. 41 (1916), pp. 449-453.

Ichimura, K., Oh, S. K. and Nakagawa, M., Light-driven motion of liquids on a photoresponsive surface, Science, Vol. 288, No. 5471 (2000), pp. 1624-1626.

Kato, K., Wakimoto, T. and Masuda, R., A new method to actuate a droplet on a plate by use of laser and ultrasonic oscillation, Transactions of the Japan Society of Mechanical Engineers Series B, Vol. 76, No. 772 (2013) pp. 141-148. (in Japanese)

Khoo, H. S. and Tseng, F. G., Self-directed movements of droplets on radially patterned surfaces based on self-assembled monolayers, Proceedings of 2006 International Conference on Microtechnologies in Medicine and Biology (2006), pp. 273-276.

Kobayashi, K., Takemura, K., Yokota, S. and Edamura, K., Mixing device for droplet $\mu$ TAS using electro-conjugate fluid (autonomous mixing of multiple droplets), Proceedings on Autumn Conference of Japan Fluid Power System Society (2013), pp. 121-123. (in Japanese)

Kobayashi, K., Kurashina, Y., Takemura, K., Yokota, S. and Edamura ,K., Mixing device for droplet $\mu$ TAS using electro-conjugate fluid, Transactions of the Japan Society of Mechanical Engineers, Vol. 80, No. 819 (2014), Paper No. 14-00288. (in Japanese)

Kumazaki, S., Hasegawa, M., Ghoneim, M., Shimizu, Y., Okamoto, K., Nishiyama, M., Oh-oka, H. and Terazima, M., A line- scanning semi-confocal multi-photon fluorescence microscope with a simultaneous broadband spectral acquisition and its application to the study of the thylakoid membrane of a cyanobacterium Anabaena PCC7120, Journal of Microscopy, Vol. 228 (2007), pp. 240-254.

Lehmann, U., Hadjidj, S., Parashar, V. K., Vandevyver, C., Rida, A. and Gijs, M. A. M., Droplet-based DNA purification in a magnetic Lab-on-a-Chip, Sensors and Actuators B: Chemical, Vol. 117 (2006), pp. 457-463.

Li, J., Chen, L., Du, L. and Li, M., Cage the firefly luciferin! - a strategy for developing bioluminescent probes, Chemical Society Reviews, Vol. 42 (2013), pp. 662-676.

McElroy, W. D., The energy source for bioluminescence in an isolated system, Proceedings of the National Academy of Sciences USA, Vol. 33 (1947), pp. 342-345.

Mochizuki, T., Wang, Y., Yamada, N. and Akiyama, H., Measurement on color change of firefly bioluminescence on temperature, Proceedings of $68^{\text {th }}$ Annual General Meeting of the Physical Society of Japan (2013), Paper No. 28pPSA-35.

Mori, K., Yamamoto, H., Takemura, K., Yokota, S. and Edamura, K., Dominant factors inducing electro-conjugate fluid flow, Sensors and Actuators A: Physical, Vol, 167 (2011), pp. 84-90.

Nakamura, R., Mizuno, Y., and Nakamura, K., Demonstration of noncontact ultrasonic mixing droplets, Japanese Journal of Applied Physics, Vol. 52 (2013), Paper No. 07 HE02.

Shimomura, O., Bioluminescence: chemical principles and methods, World Scientific, Singapore, Vol. 4 (2012) pp. xx-xxii.

Shoji, S., Micro total analysis system (uTAS), Electronics and Communications in Japan, Part 2, Vol. 82, No. 2 (1999) pp. 21-29.

Sudyoadsuk, T., Moonsin, P., Prachumrak, N., Namuangruk, S., Jungsuttiwong, S., Keawin, T. and Promarak, V., Carbazole dendrimers containing oligoarylfluorene cores as solution-processed hole-transporting non-doped emitters for efficient pre red, green, blue and white organic light-emitting diodes, Polymer Chemistry, No. 5 (2014), pp. 3982-3993.

Wang, Y., Zhe, J., Chung, B. and Dutta, P., A rapid magnetic particle driven micromixer, Microfluidics and Nanofluidics, Vol. 4 (2008), pp. 375-389.

Wixforth, A., Acoustically driven planar microfluidics, Superlattices and Microstructures, Vol. 33 (2003), pp. $389-396$. Yokota, S., Kozuki, H., Takemura, K. and Edamura, K., Proposal of high power DP-RE ECF micro motor with saw tooth-type electrodes, Proceedings of JSME Yamanashi district conference (2006) pp. 199-200.

Yokota. S., Sadamoto, A., Kondoh, Y., Otsubo, Y. and Edamura, K., A micro motor using electro-conjugate fluids(ECFs) - preposition of stator electrede(SE)-type micro ECF motors-, Transactions of the Japan Society of Mechanical Engineers Series C, Vol. 66, No. 642 (2000), pp. 627-633. (in Japanese) 